\title{
Mujeres haciendo Ciudades: APRENDIENDO DEL PASADO
}

\section{Zaida Muxí Martínez \\ Doctora arquitecta - Profesora agregada en el Departament d'Urbanisme i Ordenació del territori de l'Escola Tècnica Su- perior d'Arquitectura de Barcelona (DUOT-ETSAB) - Universitat Politècnica de Catalunya (UPC)}

RESUMEN: Se plantea revisar la presencia de las mujeres en la construcción física y simbólica de las ciudades. El texto desvela la presencia activa de las mujeres en las ciudades en que ha sido invisibilizada. En esta revisión se detectan factores comunes que podríamos denominar propios de las aportaciones de mujeres. Se trata de reconstruir la historia para aprender de las experiencias borradas.

Palabras clave: feminismo, género, ciudad, participación.

RESUM: Es planteja revisar la presència de les dones en la construcció física i simbòlica de les ciutats. El text desvela la presència activa de les dones en les ciutats en què ha estat invisibilitzada. Aquesta revisió detecta factors comuns que podríem denominar propis de les aportacions de les dones. Es tracta de reconstruir la història per a aprendre de les experiències esborrades.

PaRAules Clau: feminisme, gènere, ciutat, participació.

ABSTRACT: This paper reviews the presence of women in the physical and symbolic construction of cities. The text unveils their active presence in cities, a presence that has been made invisible. The review identifies common threads in what can be considered women's contributions. The aim of the paper is to reconstruct history so as to learn from these erased experiences.

KEYWORDS: feminism, gender, city, participation 
$\mathrm{L}$ a historia de los hechos públicos se ha escrito eliminando de ella las aportaciones de las mujeres; como no podía ser de otro modo, en la historia de la construcción de las ciudades también ha sido así.

Aclararé algunos puntos de partida que serán útiles para entender la posición del texto en el que se plantea una revisión introductoria de las aportaciones femeninas a la construcción de la ciudad occidental. Al hablar de construcción incorporo no sólo la acepción física de la misma, sino que las reflexiones, pensamientos y escritos forman parte, sin ninguna duda, de la construcción de la ciudad.

La historia se ha escrito desde el poder, y el poder es patriarcal. ${ }^{1} \mathrm{Si}$ se construye una escala de valores desde una experiencia, todo aquello que no entre en esa perspectiva queda desconocido, negado y olvidado. Por lo tanto, si son los valores y las experiencias de los hombres, a través del sistema patriarcal, las que establecen lo que es bueno y reseñable, la mitad de la humanidad no es considerada, no hay lugar para otros valores ni experiencias ni saberes. Esta valoración sesgada es extensiva a las historias de todos los otros o subalternos; es decir, las minorías étnicas o de otro tipo, los no-blancos, los no-ricos.

Pensar desde otro lugar entraña diversas dificultades, la primera cuestión es previa y clave para escribir desvelando el mundo de las mujeres, una ya clásica discusión desde las teorías feministas: ¿Cómo construir un nuevo discurso, con diferentes valores, si nos encontramos talladas por el patrón único de valores patriarcales, masculinos y machistas?

[...] El patriarcado no sólo es un peso, sino una máscara que tienes delante y que muchas veces te impide ver [...] las fuentes del patriarcado son las mismas que las fuentes de la historia de las mujeres; no hace falta ir a otros sitios a buscarlas, es [...] sobre todo, la capacidad de ver [...]. (Rivera Garretas, 2011)

1. Por patriarcado designamos una estructura social jerárquica, basada en un conjunto de ideas, prejuicios, símbolos, costumbres e incluso leyes respecto de las mujeres, por la que el género masculino domina y oprime al femenino; es una organización social profundamente discriminatoria para con las mujeres. En «El patriarcado: Una estructura invisible», $\mathrm{M}^{\mathrm{a}}$ Luisa Montero García-Celay y Mariano Nieto Navarro, http:// www.stopmachismo.net/marmar2.pdf. 
Esta situación plantea la dificultad de pensar desde fuera de la cultura y del orden simbólico (Rivera Garretas, 2003) y de encontrar nuevos indicios que permitan construir nuevos relatos.

Escribir sobre mujeres y ciudades plantea, además, describir aportaciones realizadas desde el lugar erróneo, si atendemos a la organización de los roles de género. Dado que los roles de género como construcción social constituyen una división dual y artificial según la cual a las mujeres se nos ha asignado un espacio, el interior, y un mundo de trabajo, el reproductivo, la invisibilidad de lo privado y el no-reconocimiento del valor de la reproducción, que implica todas aquellas actividades del cuidado de los otros componentes de la familia, del cuidado del hogar, de la nutrición, han marcado y marcan las actividades realizadas por el género femenino, aún hoy mayoritariamente responsabilidad y obligación de las mujeres. A la responsabilidad reproductiva se suma la productiva, en la que las mujeres han estado y están tan presentes como los hombres. Este papel difícilmente eludible para las mujeres ha tenido como consecuencia, por un lado, que no se visibilicen las actividades de las mujeres en los ámbitos de la producción, ya que no nos pertenecen, no nos son propios ni adecuados y, por otro, la invisibilidad y la no-valoración de las tareas domésticas o de la reproducción ha vaciado de contenido remarcable las aportaciones de las mujeres a la sociedad desde ese rol asignado, obligado y menospreciado.

La ciudad, su construcción y sus espacios, como representación máxima de lo público, ha sido espacio vedado para las mujeres, o eso es lo que el relato predominante de la historia nos ha hecho creer.

A modo de demostración de cómo las mujeres han estado y están presentes en la acción pública de la construcción de la ciudad me remontaré a dos situaciones casi contemporáneas, en la Edad Media, cuando grupos de mujeres se unieron para convivir y, a principios del Renacimiento, cuando una mujer con su pluma creó una ciudad, formulando en ambos casos alternativas a los modelos vigentes.

La primera situación se refiere a grupos de mujeres que a partir del siglo XIII en Europa septentrional se organizaron para constituirse en sociedades independientes que construyeron sus propios espacios y que ofrecieron a las muje- 
res una manera alternativa de vivir respecto de las opciones de la época: el matrimonio o el convento.

Estas sociedades de beguinas, o beguinajes, eran fundadas por ricas viudas o herederas que establecían un sistema urbano independiente, que se autorregulaba y autoabastecía. En 1566 llegaron a registrarse casi trescientas (Simons, 2001). El hecho de que estos recintos contuvieran edificios de culto en su interior llevó a que se los explicara como conventos, cuando en realidad se conformaban como sociedades civiles independientes que propugnaban otra manera de ser mujer: ni madre, ni esposa, ni monja o esposa de Jesús, sino trabajadoras y estudiosas. Entre sus temas de estudio se encontraba la lectura de los textos sagrados, quitando así parte de poder a la Iglesia y a los hombres que, algunos en su condición de párrocos, eran los únicos autorizados a leer y trasmitir ese conocimiento desde su propia interpretación. Precisamente esta interpretación como instrumento de poder fue cuestionada por estas mujeres, las cuales defiendieron su capacidad de leer e interpretar y de organizarse de modo alternativo. Entre otras cosas, lo que desdice la interpretación de los beguinajes como conventos es que, si el interés hubiera sido el de vivir en un convento, nada se lo hubiera impedido, podían tanto acudir a uno como fundarlo. La organización cotidiana se basaba en que las tareas del cuidado se realizaban de manera comunitaria y profesionalizada, tanto para las propias beguinas como para personas externas que acudían a vivir bajo sus cuidados; también se desplazaban para trabajar fuera cuidando personas ancianas o enfermas, así como para desarrollar labores fabriles, especialmente como tejedoras y bordadoras.

Algunos beguinajes llegaron a constituirse en verdaderas ciudadelas en el borde de la ciudad medieval. Estaban conformadas por varios edificios organizados en calles y plazas, y rodeadas de muros o fosos, aunque eran espacios abiertos a la comunidad al ser sede de numerosos servicios: desde centro de instrucción para jóvenes mujeres a centros de caridad para pobres, ancianos y enfermos.

Y la segunda situación la encontramos en el Renacimiento, cuando la construcción literaria definió a la mujer a través de los romances de pretendidos aires filosóficos, religiosos y científicos como el ser-objeto más devastador. Ante estos excesos misóginos, se levantó especialmente la voz escrita de una 
mujer, Christine de Pizan (Venecia, 1364 - Poissy, 1430), quien intervendría en la Querella de las Mujeres; especialmente, en el debate en torno al Libro de la rosa que influyó en la construcción de la misoginia europea.

En su obra más reconocida, La ciudad de las Damas (De Pizan, 14051998), habla en primera persona de la experiencia de ser mujer, su discurso constituye un cambio de perspectiva histórica. En el primer capítulo explica por qué y para qué es necesario escribir ese libro, se pregunta azorada cuáles son las razones que llevan a los hombres a inventar la figura de la mujer con ese desprecio. Explica que, a pesar de las diferentes posiciones satíricas, filosóficas o poéticas de los autores, todos confluyen en una misma conclusión: el comportamiento de las mujeres está desviado y lleno de todos los vicios; no da crédito a que tantos hombres importantes se hayan equivocado y duda de su propia experiencia de la realidad y de su propio conocimiento sobre las mujeres. Para construir el libro busca las voces y pensamientos de mujeres ejemplares, tanto reales como míticas, con quienes a través del diálogo realiza una construcción simbólica basándose en las virtudes de la razón, el buen comportamiento y la justicia de las mujeres para contrarrestar los ataques a los que son sometidas las mujeres desde la literatura y los púlpitos.

A través de La ciudad de las Damas busca romper con el silencio público que se les imponía a las mujeres con una educación en favor de un modelo de género femenino de sumisión y de privación. Su ciudad de las damas será un espacio de libertad para las mujeres, una ciudad que huirá de la destrucción y de las guerras.

Por lo tanto, hija, el privilegio de las mujeres ha sido otorgado a ti para establecer y construir la ciudad de las damas. Para su fundación y la finalización de esta ciudad sacarás las aguas dulces de nosotras y de fuentes claras, y te traeremos suficiente piedra para la construcción, más fuerte y más duradera que lo que cualquier mármol con cemento podría llegar a ser. Así, tu ciudad será muy bella, sin igual, y de duración perpetua en el mundo [...]. (De Pizan, 1405-1998)

En estas dos experiencias encontramos por lo menos dos características fundamentales del pensamiento y de la acción femenina sobre la ciudad. La primera es la duda, basada en la experiencia propia, sobre lo dado por cierto y único, de manera que se pueda generar una transformación del conocimiento. Y la segunda, la propuesta de sociedades de colaboración y no de competencia. 


\section{Siglos más tarde}

Ya en la modernidad podemos referirnos a Flora Tristán (1803-1844) como la persona pionera en denunciar la situación en la que vivían las clases obreras en el Londres victoriano, en hacer visible lo invisible. Entre sus libros se encuentra Paseos por Londres, de 1840, en el que realiza una crítica feroz a la situación de las personas de la clase obrera inglesa y describe con gran conocimiento escenas de la vida diaria. Su trabajo se adelanta a los trabajos críticos de Marx y de Engels, especialmente al de este último en La situación de la clase trabajadora en Inglaterra, de 1845.

Si bien las aportaciones de Flora Tristán son críticas respecto a los espacios urbanos, sus propuestas de acción no tienen que ver con la ciudad, sino con las políticas, la organización social y los derechos de los trabajadores y de las trabajadoras. La mirada de Flora Tristán desvela esa atención de las mujeres por los otros y por las partes más olvidadas de las ciudades.

En esta línea, en la segunda mitad del siglo XIX y principios del XX, grupos de mujeres en Inglaterra y en los Estados Unidos de América se unen para trabajar por la mejora de las zonas olvidadas de las ciudades. Sus propuestas y actividades son muchas veces despreciadas por considerarse erróneamente como acciones de beneficencia. Diría que se trataron de acciones realizadas desde el lugar asignado a las mujeres, por lo tanto, ajeno al lugar de lo público y la política.

En la sociedad mayoritariamente conservadora del siglo XIX, las mujeres encontraban grandes dificultades para acceder a roles públicos. Sin embargo, han existido algunas mujeres, que por su situación económica y social, lograron romper el cerco y actuaron para modificar cuestiones que consideraban intolerables: la situación de la vivienda, el estado de la ciudad y la falta de acceso a la educación de las mujeres.

En general, las mujeres reformistas (Ravetz, 1989) se aproximaron al problema de la vivienda y de la ciudad de manera integrada y global: desde la comprensión de las dificultades cotidianas, de las necesidades y capacidades específicas de las poblaciones, para quienes trabajaron, propusieron mejoras progresivas y realistas que se basaban en el conocimiento de la situación y en las relaciones personales. Las tres primeras, en esta lista de activistas, que 
en muchas ocasiones han sido consideradas con un cierto menosprecio como benefactoras, son Angela Burdett-Coutts (1814-1906), Octavia Hill (18381912) y Henrietta Barnett (1851-1936). Estas mujeres abrieron con sus trabajos nuevos caminos y maneras de entender y de actuar en la ciudad.

Octavia Hill será una gran activista desde muy joven. Su vida personal le lleva conocer a temprana edad las dificultades de la clase obrera, desde los quince años realiza trabajos de formación para niños y niñas pobres. Este trabajo lo realizaba en la Ladies' Cooperative Guild, una iniciativa socialista cristiana. En este entorno formó su personalidad desde muy joven; escuchaba conferencias de Charles Kingsley, Thomas Hughes y John Ruskin, entre otros, de las que obtendría enseñanzas y relaciones importantes para el posterior desarrollo de sus ideas.

Ella era contraria a la destrucción de las casas habitadas y abogaba por una mejora progresiva de las condiciones de las mismas en función de las propias capacidades familiares. Podemos considerarla una precursora de la rehabilitación tanto de barrios como de slums (asentamientos de chabolas). En 1864, le llega la primera ocasión para aplicar sus ideas sobre cómo mejorar las condiciones de vida y las viviendas de las familias obreras. A través de un préstamo que le hace John Ruskin, compra un edificio en Paradise Place, cercano a Marylebone High Street.

El sistema que ella propuso, y que llevó a la práctica con un grupo de mujeres, consistió en la mejora de las condiciones de vida en las casas de renta donde malvivían las familias obreras. Una vez se hacían con la propiedad de los inmuebles realizaban una revisión de los edificios, en la que incluían tanto los aspectos constructivos como el uso que se daba a los espacios, quiénes vivían y cómo. Si era posible técnicamente, se proponía a las familias una mejora progresiva de las condiciones constructivas e higiénicas, que podía incluir ampliaciones, a cambio del compromiso con el pago de la renta, de acuerdo con las posibilidades de cada familia. Las primeras visitas técnicas tenían también como objetivo establecer una relación de conocimiento y confianza entre las personas que habitaban y el nuevo grupo gestor. Esta relación se mantenía a lo largo del tiempo, ya que el cobro semanal de la renta se hacía acudiendo a las viviendas, lo que servía para mantener la relación, conocer los problemas de primera mano y transmitir conocimientos sobre cuestio- 
nes de higiene, sanidad, educación y nutrición a las mujeres de los edificios. El dinero recuperado con la renta se invertía en mejorar las condiciones del entorno, en realizar espacios para juegos infantiles, en ofrecer educación o en nuevos proyectos de mejora en las viviendas. Como ella misma describiera en la publicación de 1877, District Visiting: «Nuestro ideal debe ser promover la feliz interrelación entre vecinos - conocimiento mutuo, ayuda mutua...».

En 1874 ya tenía quince edificios de viviendas con 3.000 inquilinos (Morrell, 1996) y debido a esta experiencia se le ofreció, en 1884, la gestión de un gran stock de propiedades eclesiásticas, lo que aceptó con la condición de poder realizar mejoras considerables y que las voces de los inquilinos fueran escuchadas.

El hecho de trabajar con propiedades de otros hizo que incorporara a su gestión bloques, a pesar de ser una gran crítica de esta tipología. Solamente los aceptaba si ya estaban construidos y si estaban situados en una área urbana. $\mathrm{Su}$ argumento de oposición a los bloques se basaba en los siguientes criterios principales: su construcción y, ciertamente, su mantenimiento, no eran más económicos que una vivienda más vernácula; las áreas comunes eran propensas a ser foco de problemas, especialmente ligados a comportamientos, y al orden y limpieza debido, en general, a la gran densidad de habitantes; generalmente feos y sin ningún interés para ser observados, no permiten la individualización de la vivienda familiar y no hay posibilidad para adiciones o posteriores desarrollos, y había pocas posibilidades de que la gente lo sintiera suyo (Darley, 1990).

También construyó nuevas viviendas para familias obreras que cumplían con unas condiciones estéticas, arquitectónicas y de servicios ligadas a la imagen de los suburbios jardín, es decir, baja altura, densidad media, espacios verdes y equipamientos compartidos. Consideraba que las viviendas obreras debían alejarse de los espacios alienantes derivados de la monotonía y repetición de las casas en hilera, derivadas a su vez de la ordenanza de Street bylaw, o de los bloques igualmente impersonales que se construían para estas familias. Entendía el valor de la calidad del espacio en la conformación de grupos sociales y la importancia del afecto y del apego por el lugar donde se vive.

Uno de sus trabajos más reconocidos es el Red Cross Garden de 1887, según ella: «un espacio de descanso al aire libre para los cansados habitantes 
de Southwark». También construyó, adjunto al jardín, un conjunto de seis viviendas modelo y una sala comunitaria, conocida entonces como Red Cross Hall. El proyecto de este conjunto fue realizado por Elijah Hoole, quien también fue responsable, en 1884, del Toynbee Hall y del Bermondsey Settlement, en 1890. La sala comunitaria ofrecía biblioteca, espacios para el club de hombres trabajadores, grupos de mujeres, conciertos y obras de teatro, lecturas de poesía y gimnasia. Actualmente, este edificio recibe el nombre de Bishop's Hall, un nombre que no refiere a la historia del lugar y que genera una falsa interpretación del origen del edificio; la eliminación de la referencia a Octavia Hill tampoco es un hecho menor. Es así, con este tipo de borrados y renombrados, como las mujeres dejamos menos trazas en la historia.

El trabajo que lideró Octavia Hill no se limitó a la vivienda, sino que abogó por que en las ciudades existieran espacios de juegos para niños y niñas, era una gran opositora del suburbio y de la destrucción del paisaje, consideraba una necesidad que las ciudades estuvieran rodeadas, a distancias cercanas, de naturaleza para servir de espacios de esparcimiento y encuentro y, por ello, favoreció el acceso de las clases obreras a la belleza de los espacios naturales.

Fue parte de la Kyrle Society, que defendió la preservación de enclaves naturales y la defensa del patrimonio común, que sería la base del National Trust. Abogó por actuaciones de proximidad y era contraria a las grandes intervenciones realizadas por la administración. Sin embargo, su manera de trabajar, organizar y gestionar fue la base de la actuación del Gobierno británico entre las dos guerras mundiales.

Esta manera de actuar atenta a la realidad, a las necesidades de diferentes grupos y a las propias capacidades, es característica también de otros grupos de mujeres que se organizaron a finales del siglo XIX y principios del siglo XX en las principales ciudades de la costa este de los Estados Unidos de América.

Estas mujeres reformistas eran muy críticas con las acciones de los gobiernos municipales y coinciden en denunciar que la pobreza no es un problema personal, sino un problema del sistema. Su presencia pública la justifican bajo una estratagema de propiedad transitiva, la estrategia retórica del Municipal Housekeeping: el servicio de las mujeres al Estado será mejor aceptado y entendido si se relaciona con la maternidad, definiendo la ciudad como la 
extensión de sus espacios privados y, por lo tanto, de las actividades del cuidado que realizan en el hogar (Spain, 2001).

El trabajo de los grupos de Municipal Housekeeping se basaba en que las condiciones de vida en las viviendas han de ser complementada por un entorno urbano adecuado y, cuando las primeras fallan, es fundamental que desde el ámbito público, desde la ciudad, se procuren unas mejores condiciones. Asumiendo que, si su responsabilidad familiar era el mantenimiento del hogar, podían traspasar esta responsabilidad al espacio público de la ciudad, «nosotras somos las encargadas de la limpieza de nuestra casa y de que nuestra casa sea confortable y acogedora y de que estén bien los enfermos y que esté bien todo el mundo, vamos a hacer lo mismo con la ciudad» (Spain, 2001).

Chicago es una de las ciudades donde confluyen dos de los movimientos precursores en la mejora de la vida en la ciudad, el Settlement Movement (Davis, 1991) y el Municipal Housekeeping (Spain, 2001). Chicago, como lo era Londres, es a finales del siglo XIX una ciudad modélica, en la que en poco tiempo se realizarían dos importantes proyectos de mejora urbana, la Exposición Colombina de 1893, con la creación de un parque urbano a orillas del lago Michigan, y el Plan de Chicago, en 1909, de Daniel Burham y Edward Bennett. Chicago acomete grandes obras con la ambición de crear una ciudad como París en las praderas americanas, una ciudad de amplios bulevares y perspectivas monumentales, parques estratégicamente colocados e interconectados y edificios de vanguardia. Sin embargo, se habían olvidado, en la línea de la denuncia que hacía Flora Tristán, de la vida de las personas con menos recursos.

La presencia de las mujeres en los ámbitos públicos eclosiona con los reclamos sufragistas que se intensifican en el último cuarto del siglo XIX y principios del $\mathrm{xx}$, y en este tomar las calles, las mujeres proponen su presencia en la organización de la ciudad. Por un lado, las mujeres de los Settlement Movements que se dedican a trabajar en los barrios más pobres, visitan las casas y colaboran en la mejora de la higiene y la alimentación de las familias que allí viven.

La ciudad, más allá de los espacios públicos y edificios emblemáticos, está sucia y abandonada. Encuentran inaceptable que en las calles donde jue- 
gan y pasan el día los niños más pobres se acumulen agua y suciedad, hasta tal punto que los animales muertos quedan allí abandonados durante días. Según explica Daphne Spain, las calles llenas de basura y el ruido se consideraban temas menores frente a la amenaza más importante de la calidad del aire y la contaminación del agua, que eran los principales problemas en las ciudades. Además de aire contaminado, el agua sucia era también un factor de preocupación. El cólera era una amenaza constante, y sólo respirar significaba una experiencia desagradable para los que sobrevivían a la epidemia.

Las asociaciones del Municipal Housekeeping fomentan la construcción de baños públicos para la higiene de las familias, ya que el agua corriente y los baños eran un lujo de pocas de ellas. Para tener una ciudad limpia y sana, había que comenzar por facilitar la limpieza de sus habitantes.

A propuesta de estas organizaciones femeninas se gestionó la mejora de los espacios públicos cotidianos. Dirigieron la limpieza de las calles, organizaron escuelas maternales para que los niños y las niñas no estuviesen en las calles cuando sus padres fueran a trabajar y para garantizarles al menos un desayuno.

Las mujeres del Municipal Housekeeping proponen, ante la falta de espacio libre en la ciudad industrial, la utilización temporal de solares abandonados para transformarlos en pequeños espacios de juegos infantiles. La propuesta deriva de la observación de la falta de espacios públicos de calidad en los barrios pobres, y de los solares abandonados y llenos de basura que aumentaban las condiciones de insalubridad.

A partir de estas experiencias se forman asociaciones para espacios de juegos infantiles — en los que no se buscaba la gran belleza, sino el espacio limpio y adecuado para el esparcimiento- cercanos a las residencias. La asociación precursora del movimiento de los patios de juegos fue fundada en Boston, en 1880 por la doctora Marie Zakrzewska, quien creó los primeros «jardines de arena», espacios para el juego bajo el control de una mujer respetable. En 1906 se formará la Playground Association of America, que coexiste con el movimiento City Beautiful. Este último abogaba por grandes espacios verdes, bellamente diseñados, con monumentos, y temía que los patios de juego le quitaran presupuestos. Sin embargo, las grandes superficies eran muy caras y no servían como espacio cotidiano de recreo, que era lo que 
buscaba la asociación. Esta pugna ejemplifica dos miradas opuestas sobre la ciudad, la de vivir y la de exhibir, la de la gente y la de los agentes. Estos usos temporales como espacios de juego infantil inauguran una tradición que ha reaparecido en la actualidad y que fue retomada en la crisis alimentaria de la gran depresión en ciudades como Nueva York - para crear huertos - o en el Ámsterdam de Jakoba Mulder en la segunda postguerra.

Jakoba (Ko) Helena Mulder (1900-1988), ingeniera graduada en 1926 en la Technische Hogeschool, comenzó a trabajar en 1930 en el Departamento de Planificación Urbana de la ciudad de Ámsterdam. Ese mismo año proyectó el trabajo que le dio fama entre la ciudadanía: el Boschplan, actual bosque de Ámsterdam (Amsterdamse Bos). Este proyecto fue al tiempo un espacio medioambientalmente precursor y una estrategia para mejorar la ciudad, generando una política de empleo en un momento de depresión económica.

En 1958 Jakoba Mulder sucedió a Cornelis van Eesteren como directora de planeamiento de Ámsterdam (anteriormente había sido subdirectora). El equipo del que ambos formaban parte redactó, entre las dos guerras mundiales, el plan urbano holandés más famoso, que fue la extensión de Ámsterdam. $\mathrm{Su}$ importancia queda reflejada en la escala del plan que permitía a la ciudad crecer de 750.000 habitantes, en 1930, a cerca de un millón al final del siglo. El plan fijó las bases para el crecimiento de los llamados pueblos jardín (tuinsteden) como Slotermeer. Después de 1945 fue aplicado mucho más rápido de lo previsto, y según Ward (2002), ha de ser considerado uno de los planes más exitosos de los esfuerzos de entreguerras para las grandes ciudades europeas. Para Ward, este plan marca una tendencia sumamente interesante en la evolución profesional del planeamiento, y es que se trata del primer plan en ser conscientemente preparado y presentado como un trabajo de equipo. Para el plan fueron sumamente importantes tanto el trabajo de investigación de Theo van Lohuizen como el de planificación de Jakoba Mulder.

Desde el comienzo de su carrera le preocupó la falta de lugares adecuados para el juego de las niñas y de los niños en la ciudad. Se preguntaba a qué se debía su ausencia, siendo algo tan fácil de conseguir. Su atención a la realidad, y a los pequeños detalles, en escala, pero de gran influencia en la calidad de vida de las personas, le llevó en 1947, a proponer un 
sistema bottom-up (ascendente) de creación de espacios públicos para el juego infantil. Jakoba Helena Mulder había observado desde su ventana a una niña jugando en un rincón de la calle sin más recursos que imaginación, tierra y una lata, y se le ocurrió que era posible crear pequeños y económicos espacios llenos de posibilidades para el juego infantil, para cuyo diseño se ofreció voluntario el joven Aldo van Eyck. El mecanismo propuesto para crear estos espacios era siempre que una persona o un grupo de ciudadanos que conociesen las necesidades concretas de la población, identificara y propusiera solares vacíos, esquinas, aceras y otros pequeños terrenos urbanos subutilizados para transformarlos en uno de estos espacios de juego, a lo que el ayuntamiento respondía, una vez comprobada su viabilidad, con un diseño específico. Con este sistema se crearon 700 parques infantiles entre 1948 y 1978, esparcidos por toda la ciudad de Ámsterdam, y que Aldo van Eyck tuvo la oportunidad de proyectar desde su empleo en la Oficina de Trabajos Públicos de la ciudad. Una serie de elementos simples permitían conformar espacios de juegos adecuados a cada superficie, cada uno resultaba diferente y, lo que es más importante, con una gran variedad de formas, texturas y posibilidades capaces de provocar la utilización creativa de dichos elementos por los niños y niñas. (Montaner y Muxí, 2011).

\section{Conclusión: ¿qué aprendemos de estas experiencias?}

En estas experiencias encontramos características comunes que son hoy visibles aún en las propuestas contemporáneas realizadas desde los feminismos y con perspectiva de género. Fundamentalmente, consisten en que no se puede actuar sin un conocimiento profundo y cercano de la realidad que permita hacerlo de manera más adecuada a cada micro-realidad, alejándose del neutro universal que enmascara la experiencia jerárquica y masculina del patriarcado. Y, por otro lado, la colaboración y la cooperación entre agentes muy diversos en estructuras que tienden más a la horizontalidad que a una pirámide jerárquica. 


\section{Referencias}

DARLey, G. (1990): Octavia Hill. A Life, Constable, London.

DAvis, A. F. (1991): The Social Settlements and the Progressive Movement 1890-1914, New Brinswick, Rutgers University Press, New Jersey.

De Pizan, C. (1998): The book of the City of Ladies, Persea, New York. [Le livre de la cité des Dames. 1st edition 1405.]

Montaner, J. M. y Z. Muxí (2011): Arquitectura y política. Ensayos para mundos alternativos, Gustavo Gili, Barcelona.

Ravetz, A. (1989): «A View from the Interior», en Attfield, J. \& P. Kikham, Eds. $A$ View from the Interior. Feminism, Women and Design, Women's Press, London.

Rivera Garretas, M-M. (2003): Nombrar el mundo en femenino. Pensamiento de las mujeres y teoría feminista, Icaria ( $1^{\text {a }}$ edición 1994), Barcelona.

- (2011): «La memoria de las mujeres» en Mujer Pública, $n^{\circ} 4$, Revista de discusión feminista. Coordinación: Mujeres Creando, Idoia Romano, María Galindo, La Paz.

Simons, W. (2001): Cities of Ladies. Beguine Communities in the Medieval Low Countries. 1200-1565, University of Pennsylvania Press, Philadelphia.

SpaIn, D. (2001): How Women Saved the City, University of Minnesota Press, Minneapolis-London. 